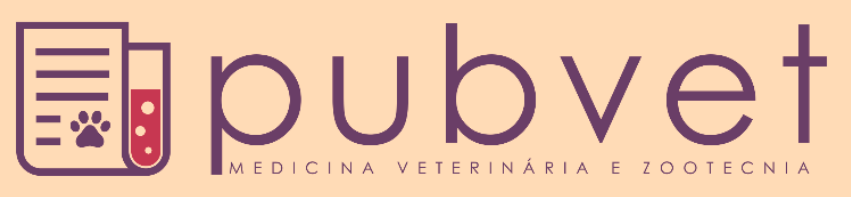

https://doi.org/10.31533/pubvet.v14n6a593.1-5

\title{
Fisioterapia neurofuncional na meningoencefalomielite granulomatosa cervical: relato de caso
}

\author{
Daniela Loureiro Henrique ${ }^{10 *}$, Beatriz Ribeiro $\operatorname{Gaspar}^{2} \theta$, Cintia Ghorayeb ${ }^{3} \theta$, Felipe Andrei \\ Soarez Abreu ${ }^{3}$, Amanda Gomes ${ }^{4}$
}

${ }^{1}$ Mestranda em Medicina Veterinária e Bem-Estar Animal Santo Amaro (UNISA). São Paulo-SP Brazil.

${ }^{2}$ Discente na Universidade de São Paulo (USP). São Paulo-SP Brazil

${ }^{3}$ Médico Veterinário Autônomo

${ }^{4}$ Docente da Faculdade das Américas (FAM). São Paulo-SP Brazil

*Autor para correspondência, E-mail: daniela@pataepatela.com.br

Resumo. A Fisioterapia Neurofuncional é uma área extremamente importante na fisioterapia humana e vastamente conhecida. O foco principal é a realização de ações básicas pelo paciente neurologicamente comprometido como ficar em estação, pegar um copo e/ou caminhar. Na fisioterapia veterinária é uma vertente nova, porém em franca ascensão. A Meningocencefalite Granulomatosa (MEG) é uma doença comum na clínica veterinária, de prognóstico reservado a ruim e que provoca alterações neurológicas importantes no paciente. Embora seja uma doença grave e progressiva, a fisioterapia neurofuncional na MEG foi importante para o retorno de funções básicas como o caminhar, além de ajudar no controle de dor causada pela espasticidade apresentada pela paciente. É necessário que novos estudos sejam realizados, mas, no que se refere à qualidade de vida, ela se mostrou muito eficiente.

Palavras chave: canina, granulomatosa, meningoencefalite, neurofuncional, reabilitação

\section{Neurofunctional physiotherapy in cervical granulomatous meningoencephalomyelitis: case report}

\begin{abstract}
Neurofunctional Physiotherapy is an extremely important and widely known area in human physiotherapy. The main focus is the performance of basic actions by the neurologically compromised patient such as staying in station, taking a glass and / or walking. In veterinary physiotherapy it is a new aspect, but it is on the rise. Granulomatous Meningocencephalitis (MEG) is a common disease in the veterinary clinic, with a poor prognosis and that causes important neurological changes in the patient. Although it is a serious and progressive disease, neurofunctional physical therapy in MEG was important for the return of basic functions such as walking, in addition to helping to control pain caused by the spasticity presented by the patient. It is necessary that further studies are carried out, but with regard to quality of life, it has proved to be very efficient.
\end{abstract}

Keywords: canine, granulomatous, meningoencephalitis, neurofunctional, rehabilitation

\section{Fisioterapia neurofuncional en meningoencefalomico cervical granulomatoso: informe de un caso}

Resumen. La fisioterapia neurofuncional es un área extremadamente importante y ampliamente conocida en fisioterapia humana. El enfoque principal es la realización de acciones básicas por parte del paciente neurológicamente comprometido, como permanecer en la estación, tomar un vaso y / o caminar. En la fisioterapia veterinaria es un aspecto 
nuevo, pero está en aumento. La meningocenfalitis granulomatosa (MEG) es una enfermedad común en la clínica veterinaria, con mal pronóstico y que causa cambios neurológicos importantes en el paciente. Aunque es una enfermedad grave y progresiva, la fisioterapia neurofuncional en MEG fue importante para el retorno de funciones básicas como caminar, además de ayudar a controlar el dolor causado por la espasticidad presentada por el paciente. Es necesario que se realicen más estudios, pero con respecto a la calidad de vida, ha demostrado ser muy eficiente.

Palabras clave: canina, granulomatosa, meningoencefalitis, neurofuncional, rehabilitación

\section{Introdução}

A Meningoencefalite Granulomatosa (MEG) é uma doença inflamatória, progressiva, de causa indefinida, com localização no Sistema Nervoso Central (SNC) que afeta, especialmente, substância branca, cerebelo, porção caudal do tronco cerebral e medula espinhal cervical; provocando sinais clínicos que podem variar de convulsões, hemiparesia, deficiências visuais ou até mesmo óbito (Crespo, 2014; Dewey \& Costa, 2016). A afecção pode ser focal, multifocal, forma mais comum; e ocular, manifestação mais rara (Dewey \& Costa, 2016). O tratamento padrão utilizado é terapia imunossupressora com glicocorticoides; porém, já existem vários protocolos com outros imunossupressores sendo utilizados com sucesso (Dewey \& Costa, 2016). Em geral, o prognóstico da doença é de reservado a ruim. Contudo, a introdução de novos medicamentos associada à fisioterapia neuro funcional vem melhorando a qualidade de vida além de aumentar a sobrevida desses pacientes (Clark-Wilson et al., 2014; Dewey \& Costa, 2016).

Embora a Fisioterapia Neuro funcional em humanos data desde os anos 1980, na Medicina Veterinária a mesma ainda é incipiente. Tem como objetivo incorporar aprendizagem de habilidades motoras e promover o desenvolvimento de competências, de forma rotineira, em atividades práticas que são necessárias para a vida cotidiana; minimizando os efeitos da doença no paciente, mantendo as habilidades funcionais e melhorando algumas condições físicas ainda preservadas (Guimarães et al., 2016).

O presente trabalho descreve a aplicação de técnicas fisioterápicas como parte dos cuidados paliativos em um cão acometido por MEG, bem como os resultados observados.

\section{Relato de caso}

A paciente canina, da raça Maltês de três anos e 4,3 kg, foi atendida pela fisiatra veterinária após o adequado diagnóstico - por ressonância magnética - e controle medicamentoso do quadro agudo de MEG focal. Na primeira avaliação, o animal encontrava-se em tetraparesia espástica não deambulatória, com propriocepção diminuída e aumento do tônus muscular nos 4 membros, sendo a propriocepção ausente em membro torácico direito (MTD). Associadamente, havia hiperreflexia patelar bilateral; ausência do reflexo flexor e de dor superficial no MTD - que se apresentava espástico em contra extensão.

O tratamento fisioterápico foi dividido em duas fases e foi realizado três vezes por semana durante 60 dias. Na primeira fase, houve foco no controle de dor, mobilização articular e estímulo neuro locomotor. Para isso, foram realizadas séries de exercícios contendo 20 movimentos de bicicleta (extensão e flexão) com o MTD em cima do disco de equilíbrio, visando estímulo do reflexo flexor; em conjunto, exercício de 4 apoios em bola terapêutica foi implementado, com objetivo de manter a paciente na posição correta e em isometria, além do estímulo neuromuscular; após, foi submetida a magneto terapia em frequências de $60 \mathrm{hz}, 40 \mathrm{hz}$ e $20 \mathrm{hz}$ a cada $10 \mathrm{~min}$., totalizando $30 \mathrm{~min}$, para o adequado relaxamento muscular; e por fim, mobilização articular do MTD, iniciada no carpo e finalizada na escapulo-umeral, para manter e melhorar a lubrificação articular. A partir da resposta positiva da paciente, como apoio do MTD, deambulação sem apoio e maior amplitude de movimento na articulação escapulo-umeral direita, os exercícios tornaram-se mais complexos.

Para a segunda fase, o foco das sessões foi o fortalecimento seguido do controle do tronco, com estímulo do MTD. Foram então realizadas 5 séries de caminhada em cavalete, para incrementar a propriocepção e flexão articular; 5 séries de exercício de arrasto, trabalhando a força, equilíbrio do tronco e extensão da articulação escapulo-umeral; e por último, isometria em disco de equilíbrio durante 
5 min com membros torácicos apoiados no chão. Durante todo o tratamento, utilizou-se uma órtese proprioceptiva no MTD para que a mesma pudesse manter os dígitos na posição correta.

\section{Resultados e discussão}

Com o tratamento medicamentoso e a associação da fisioterapia, a paciente começou a deambular independentemente, apesar do MTD ainda estar em espasticidade na contra extensão e ter menor nocicepção superficial. Após 20 sessões, a mesma iniciou movimento voluntário da articulação escapulo-umeral, conseguindo posicionar mais adequadamente o membro ao passo. Além disso, a paciente apresentou melhora significativa no equilíbrio quando comparado ao início do tratamento fisioterápico. Houve também um incremento na goniometria das articulações do MTD. No início do tratamento, na articulação escapulo-umeral tanto o ângulo de extensão quanto de flexão foram de $120^{\circ}$, na úmero-radio-ulnar eram de $174^{\circ}$ respectivamente enquanto na articulação metacarpiana os ângulos foram de $180^{\circ}$, evidenciando a espasticidade do MTD. Após as 20 sessões, a melhora na flexão de todas as articulações foi bem evidente, sendo $100^{\circ}$ no ombro, $90^{\circ}$ no cotovelo e $70^{\circ}$ no carpo. Infelizmente, houve piora abrupta do quadro de MEG e a paciente foi eutanasiada.

Este relato de caso buscou avaliar como a fisioterapia veterinária pode ser benéfica quando associada ao tratamento medicamentoso de um cão afetado por meningoencefalite granulomatosa. Os resultados encontrados sugerem que as técnicas utilizadas foram efetivas em ampliar o bem-estar e qualidade de vida da paciente.

Essa afirmação pode ser justificada tanto pelo aumento da amplitude de movimento adquirida, como pela autonomia para deambular adequadamente. Esta última foi conquistada pela aplicação de diferentes técnicas, entre elas, a implementação de exercícios em que há foco na estabilização do core, como os de isometria em quatro ou dois apoios em bola/disco terapêutico (Figura 1). Estes são essenciais para a conquista de equilíbrio e proficiência motora, contribuindo para melhor deambulação do paciente (Au et al., 2014; Clayton, 2016). Essa abordagem faz parte da neuro reabilitação funcional, que considera como um de seus princípios o desenvolvimento da automação e manutenção de atividades diárias (ClarkWilson et al., 2014), como o caminhar por exemplo.

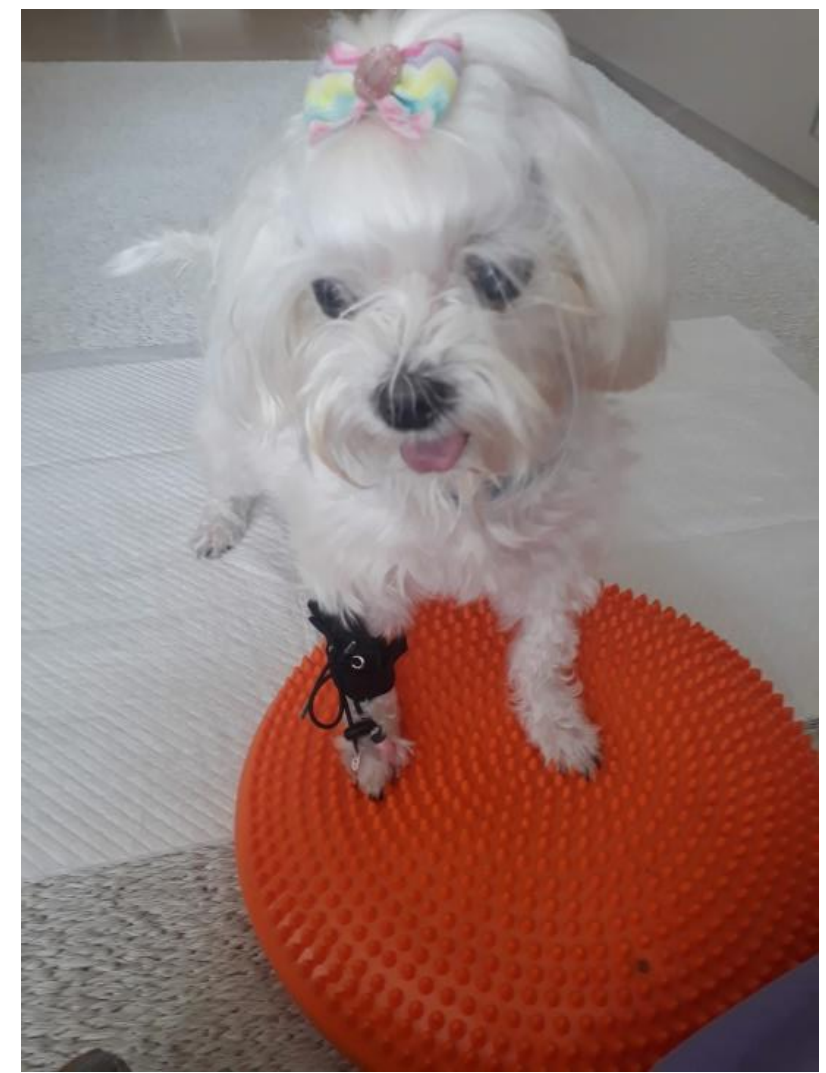

Figura 1. Paciente realizando exercício de equilíbrio no disco. Imagem gentilmente cedida pela fisiatra veterinária Daniela Loureiro Henrique. 
A partir dos alongamentos ativos e mobilização articular realizados nas sessões, foi conquistada uma maior amplitude de movimento no MTD. Este incremento está diretamente ligado com maior flexibilidade, torque articular e mobilidade funcional do membro (Batista et al., 2009) de forma a contribuir diretamente para a performance motora da paciente (Sharman et al., 2006).

A realização de exercícios foi fundamental para o aumento de tônus do core e recuperação da propriocepção e equilíbrio da paciente. $\mathrm{O}$ uso da bola terapêutica faz com que a paciente, para manter o equilíbrio, realize uma contração isométrica importante tanto do core quanto da musculatura dos membros (Henrique, 2020). Já no exercício do arrasto (Figuras 2 e 3), a exigência física é muito maior: há um componente isométrico (contração da musculatura do core) e incremento na força quando o animal faz flexão e extensão dos quatro membros ao realizar o exercício (Hamilton et al., 2004).

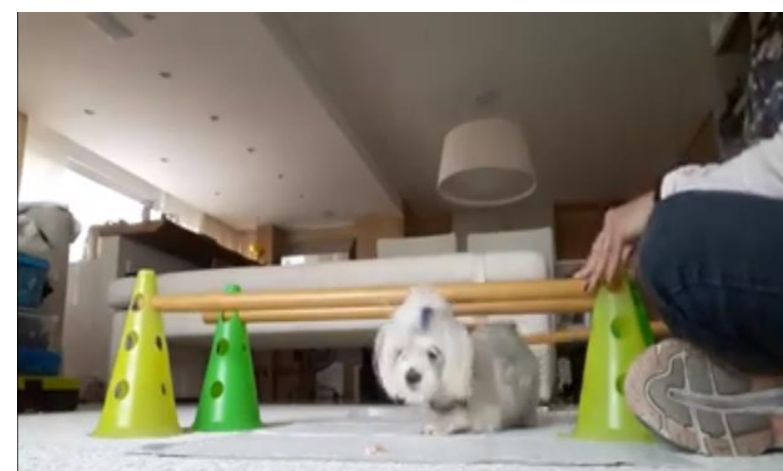

Figura 2. Início do exercício de arrasto. Imagem gentilmente cedida pela fisiatra veterinária Daniela Loureiro Henrique.

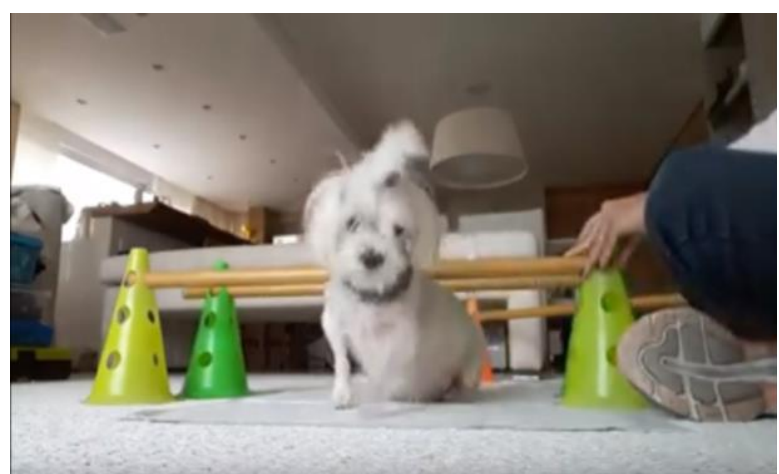

Figura 3. Finalização do exercício de arrasto. Imagem gentilmente cedida pela fisiatra veterinária Daniela Loureiro Henrique.

Na caminhada em cavaletes, além de melhorar a amplitude de movimento das articulações, ainda é estimula a propriocepção, equilíbrio e coordenação motora do animal (Henrique, 2020; Hamilton et al, 2004). Utilizou-se a magneto terapia como agente físico com intuito de aumentar a analgesia e diminuir os espasmos musculares segundo (Lewicki \& Valim, 2020).

Segundo o trabalho de revisão sistemática de Guimarães et al. (2015), conclui-se que os benefícios da fisioterapia neuro funcional para os pacientes de Esclerose Amiotrófica Lateral (ELA) foram manutenção da funcionalidade por um período maior, diminuição da espasticidade além de melhorar algumas condições físicas ainda preservadas. Já em pacientes com Mal de Parkinson (Pompeu \& Pompeu, 2001) afirmam que, embora o tratamento fisioterápico não interfira na progressão da doença, ele pode beneficiar uma melhor execução das atividades diárias. Ou seja, mesmo que não impeça o curso natural da doença, a fisioterapia neuro funcional mostrou trazer importantes benefícios para os pacientes (Pompeu \& Pompeu, 2001).

\section{Conclusão}

Ficou demonstrada evolução no quadro da paciente com a Fisioterapia Neuro funcional; porém, mais estudos devem ser realizados.

\section{Referências bibliográficas}

Au, M. K., Chan, W. M., Lee, L., Chen, T. M. K., Chau, R. M. W., \& Pang, M. Y. C. (2014). Core stability exercise is as effective as task-oriented motor training in improving motor proficiency in children with developmental coordination disorder: a randomized controlled pilot study. Clinical Rehabilitation, 28(10), 992-1003. DOI: https://doi.org/10.1177\%2F0269215514527596

Batista, L. H., Vilar, A. C., Ferreira, J. J. A., Rebelatto, J. R., \& Salvini, T. F. (2009). Active stretching improves flexibility, joint torque, and functional mobility in older women. American Journal of Physical Medicine \& Rehabilitation, 88(10), 815-822. DOI: 10.1097/PHM.0b013e3181b72149

Clark-Wilson, J., Giles, G. M., \& Baxter, D. M. (2014). Revisiting the neurofunctional approach: Conceptualizing the core components for the rehabilitation of everyday living skills. Brain Injury, 
28(13-14), 1646-1656. DOI: https://doi.org/10.3109/02699052.2014.946449

Clayton, H. M. (2016). Core training and rehabilitation in horses. Veterinary Clinics: Equine Practice, 32(1), 49-71. DOI: https://doi.org/10.1016/j.cveq.2015.12.009

Crespo, F, D.;(2014) Meningoencefalite Granulonamotosa em Cães. Monografia apresentada à Faculdade de Medicina Veterinária da Universidade Federal do Rio Grande do Sul. Porto Alegre

Dewey, C. W., \& Costa, R. C. (2016). Practical guide to canine and feline neurology (3 Ed.). Wiley Blackwell.

Guimarães, M. T. S., Vale, V. D., \& Aoki, T. (2016). The benefits of neurofunctional physiotherapy in patients with Amyotrophic Lateral Sclerosis: a systematic review. ABCS Health Sciences, 41(2), 8189. DOI: https://doi.org/10.7322/abcshs.v41i2.874

Hamilton, S., Millis, D. L., Taylor, R. A., \& Levine, D. (2004). Therapeutic Exercises. In D. L. Millis, D. Levine, \& R. A. Taylor (Eds.), Canine Rehabilitation and Physical Therapy (pp. 244-276). Sauders Elsevier.

Henrique, D. L. (2020). Exercícios terapêuticos. In F. Vituri \& D. L. Henrique (Eds.), Fisioterapia em pequenos nimais (pp. 95-102). Editora Payá.

Lewicki, V., \& Valim, M. M. (2020). Agentes físicos na reabilitação veterinária. In F. Vituri \& D. L. Henrique (Eds.), Fisioterapia em pequenos animais (pp. 69-79). Editora Payá.

Pompeu, S. M. A. A., \& Pompeu, J. E. (2001). Abordagem fisioterapêutica na doença de parkinson. In C. I. Marchese (Ed.), Fisioterapia neuro funcional: aspectos clínicos e práticos (pp. 137-152). Editora CRV. DOI: https://doi.org/10.5335/rbceh.2012.259

Sharman, M. J., Cresswell, A. G., \& Riek, S. (2006). Proprioceptive neuromuscular facilitation stretching. Sports Medicine, 36(11), 929-939. DOI: https://doi.org/10.2165/00007256-20063611000002

Recebido: 5 de abril, 2020

Aprovado: 21 de maio, 2020.

Disponível online: 14 julho, 2020.
Licenciamento: Este artigo é publicado na modalidade Acesso Aberto sob a licença Creative Commons Atribuição 4.0 (CC-BY 4.0), a qual permite uso irrestrito, distribuição, reprodução em qualquer meio, desde que $\mathrm{o}$ autor e a fonte sejam devidamente creditados. 